\title{
One-Dimensional Vertex Models Associated with a Class of Yangian Invariant Haldane-Shastry Like Spin Chains
}

\author{
Bireswar BASU-MALLICK ${ }^{\dagger}$ and Nilanjan BONDYOPADHAYA ${ }^{\ddagger}$ and Kazuhiro HIKAMI ${ }^{\text {B }}$ \\ $\dagger$ Theory Group, Saha Institute of Nuclear Physics, 1/AF Bidhan Nagar, Kolkata 700 064, India \\ E-mail: bireswar.basumallick@saha.ac.in \\ ¥ Integrated Science Education and Research Centre, Siksha-Bhavana, Visva-Bharati, \\ Santiniketan 731 235, India \\ E-mail: nilanjan.bondyopadhaya@saha.ac.in \\ $\S$ Department of Mathematics, Naruto University of Education, Tokushima 772-8502, Japan \\ E-mail: KHikami@gmail.com
}

Received September 06, 2010, in final form November 30, 2010; Published online December 10, 2010 doi:10.3842/SIGMA.2010.091

\begin{abstract}
We define a class of $Y\left(s l_{(m \mid n)}\right)$ Yangian invariant Haldane-Shastry (HS) like spin chains, by assuming that their partition functions can be written in a particular form in terms of the super Schur polynomials. Using some properties of the super Schur polynomials, we show that the partition functions of this class of spin chains are equivalent to the partition functions of a class of one-dimensional vertex models with appropriately defined energy functions. We also establish a boson-fermion duality relation for the partition functions of this class of supersymmetric HS like spin chains by using their correspondence with onedimensional vertex models.
\end{abstract}

Key words: Haldane-Shastry spin chain; vertex model; Yangian quantum group; bosonfermion duality relation

2010 Mathematics Subject Classification: 81R12; 81R50; 82B10; 82B05

\section{Introduction}

It is well known that one-dimensional (1D) quantum integrable spin chains can be divided into two classes depending on their range of interaction. One such class consists of spin chains having only local interactions like nearest or next-to-nearest neighbor interaction. These spin chains are usually solvable through coordinate or algebraic Bethe-ansatz technique [1, 2, 3]. Isotropic and anisotropic versions of Heisenberg spin chain, Hubbard model etc. are examples of this type of spin models with short range interaction. It is worth noting that, there exists a connection between such quantum spin chains and vertex models in classical statistical mechanics. More precisely, the Hamiltonians of 1D quantum spin models are related to the differentiated transfer matrices of the corresponding $2 \mathrm{D}$ vertex models in statistical mechanics $[4,5]$.

Apart from the above mentioned class of spin chains, there exist another class of 1D quantum integrable spin systems for which all constituent spins mutually interact with each other through long range forces. Haldane-Shastry (HS) spin chain $[6,7]$ and Polychronakos spin chain (also known as Polychronakos-Frahm spin chain) $[8,9,10]$ associated with the $A_{N}$ type of root system are well known examples of quantum integrable spin systems with long range interaction. This type of spin chains have attracted a lot of attention in recent years due to their exact solvability and applicability in a wide range of subjects like fractional statistics [11, 12, 13, 14], SUSY Yang-Mills theory and string theory [15, 16, 17], Yangian quantum group [18, 19, 20, 21, 22] 
etc. The lattice sites of HS spin chain are uniformly distributed on a circle and, for the case of most general $s u(m \mid n)$ supersymmetric version of this model, each lattice site is occupied by either one of the $m$ type of bosonic 'spins' or one of the $n$ type of fermionic 'spins'. The Hamiltonian of such $s u(m \mid n)$ HS spin chain is given by [23, 24]

$$
\mathcal{H}_{\mathrm{HS}}^{(m \mid n)}=\frac{1}{2} \sum_{1 \leq j<k \leq N} \frac{1+\hat{P}_{j k}^{(m \mid n)}}{\sin ^{2}\left(\xi_{j}-\xi_{k}\right)},
$$

where $\xi_{j}=j \pi / N$ and $\hat{P}_{j k}^{(m \mid n)}$ represents the supersymmetric exchange operator which interchanges the spins on the $j$-th and $k$-th lattice sites. On the other hand, the lattice sites of Polychronakos spin chain are non-uniformly distributed on a line and the Hamiltonian of the $s u(m \mid n)$ supersymmetric version of this model is given by [25, 21]

$$
\mathcal{H}_{\mathrm{P}}^{(m \mid n)}=\sum_{1 \leq j<k \leq N} \frac{1+\hat{P}_{j k}^{(m \mid n)}}{\left(x_{j}-x_{k}\right)^{2}},
$$

where $x_{j}$ 's are the zero points of the $N$-th order Hermite polynomial. It may noted that, for the special cases with either $n=0, m>1$, or $m=0, n>1,(1.1)$ and (1.2) reduce to the Hamiltonians of the corresponding nonsupersymmetric spin chains. For example, in the special case with $n=0, m>1,(1.1)$ and (1.2) reduce to the Hamiltonians of the $s u(m)$ bosonic HS and Polychronakos spin chain respectively. One of the most important feature of this type of spin chains is the embedded Yangian quantum group symmetry of their Hamiltonians, which allows one to write down the corresponding energy spectra in closed forms even for finite number of lattice sites. Consequently, the partition functions of such spin chains can be expressed through Schur polynomials associated with the irreducible representations of the Yangian quantum group $[20,21,26]$. In this context it should be noted that, for finite number of lattice sites, in general one can not analytically solve the Bethe ansatz equations associated with integrable spin chains with short range interaction and express the corresponding energy spectra in closed form $[1,2,3]$.

Since it is possible to express the spectra of the above mentioned quantum integrable spin chains with long range interactions in closed forms, one may naturally enquire whether such spectra can be generated from the energy functions associated with some classical vertex models. Indeed, it has been found earlier that the classical partition function of a $1 \mathrm{D}$ vertex model, emerging from the character of spin path configurations, is completely equivalent to the partition function of $s u(m)$ Polychronakos spin chain [27]. Moreover, the energy levels of $s u(m)$ Polychronakos spin chain exactly match (including the degeneracy factors) with the energy functions associated with this 1D vertex model. Similar equivalence has been established between $s u(m \mid n)$ supersymmetric Polychronakos spin chain and a vertex model with suitably defined energy function [28]. It is worth noting that, a recursion relation satisfied by the partition function of the Polychronakos spin chain plays a crucial role in establishing the above mentioned equivalence between quantum spin chain and classical vertex model. However, it is not yet known whether the partition function of HS spin chain would also satisfy similar type of recursion relation. Therefore, some new approach is required to search for $1 \mathrm{D}$ vertex model whose partition function would be equivalent to that of $s u(m)$ HS spin chain or its supersymmetric extension. The purpose of the present article is to develop such a general formalism, which can be used to find out the vertex models associated with a class of Yangian invariant spin chains including the HS model.

The arrangement of this article is as follows. In Section 2 we briefly describe border strips and super Schur polynomials associated with the irreducible representations of $Y\left(s l_{(m \mid n)}\right)$ Yangian algebra. In Section 3, we define a class of $s u(m \mid n)$ HS like spin chains by assuming that their partition functions can be written in a particular form in terms of the super Schur polynomials. 
Then, by using some properties of the super Schur polynomials, we show that the partition functions of this class of spin chains are equivalent to the partition functions of a class of 1D vertex models with appropriately defined energy function. Since $s u(m \mid n)$ HS and Polychronakos spin chains belong to the above mentioned class of Yangian invariant spin chains, we easily obtain the corresponding vertex models through this approach. In Section 3, we also establish a bosonfermion duality relation for the partition functions of supersymmetric HS like spin chains by using their correspondence with 1D vertex models. In Section 4, we make some concluding remarks.

\section{Border strips and super Schur polynomials}

Since in our analysis we wish to consider some HS like spin chains with embedded $Y\left(s l_{(m \mid n)}\right)$ symmetry, let us first briefly discuss about 'border strips' which represent a class of irreducible representations of the $Y\left(s l_{(m \mid n)}\right)$ algebra and span the Fock spaces of Yangian invariant spin systems $[20,21,26,27]$. For a spin system with $N$ number of lattice sites, a border strip is uniquely characterized by a set of positive integers $k_{1}, k_{2}, \ldots, k_{r}$, where $\sum_{i=1}^{r} k_{i}=N$, and $r$ is an integer which can take any value from 1 to $N$. Thus the vector $\mathbf{k} \equiv\left\{k_{1}, \ldots, k_{r}\right\}$ belongs to the set $\mathcal{P}_{N}$ of ordered partitions of $N$. Let us denote the border strip corresponding to the vector $\mathbf{k}$ by $\left\langle k_{1}, k_{2}, \ldots, k_{r}\right\rangle$, which is drawn in Fig. 1 .

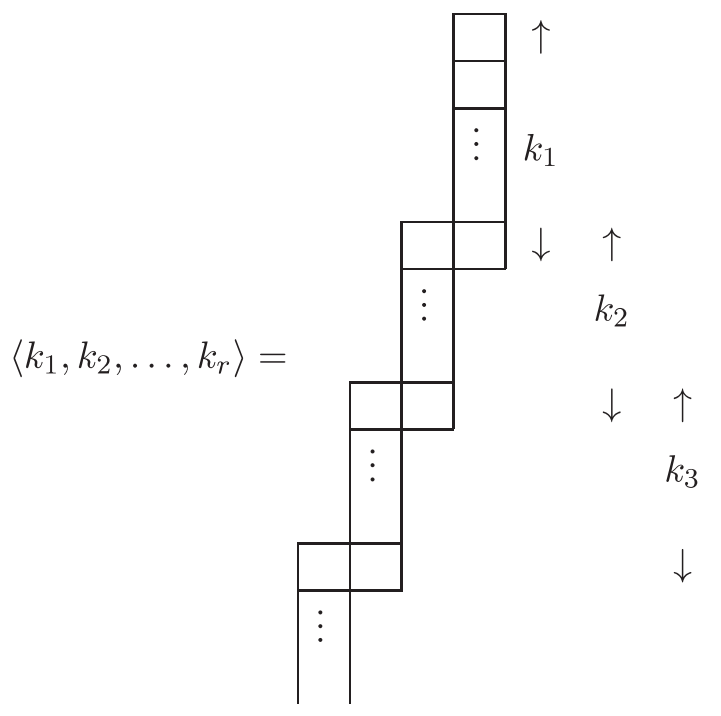

Figure 1. Shape of the border strip $\left\langle k_{1}, k_{2}, \ldots, k_{r}\right\rangle$.

The super Schur polynomial corresponding to this border strip is denoted by $S_{\left\langle k_{1}, k_{2}, \ldots, k_{r}\right\rangle}(x, y)$, where $x \equiv\left\{x_{1}, \ldots, x_{m}\right\}$ represents the bosonic variables and $y \equiv\left\{y_{1}, \ldots, y_{n}\right\}$ represents the fermionic variables. For the purpose of defining such super Schur polynomial, we set $\mathbf{B}^{(m \mid n)}=$ $\mathbf{B}_{+}^{(m)} \cup \mathbf{B}_{-}^{(n)}$, where

$$
\mathbf{B}_{+}^{(m)}=\{1,2, \ldots, m\}, \quad \mathbf{B}_{-}^{(n)}=\{m+1, m+2, \ldots, m+n\} .
$$

The Young tableaux $T$ is obtained by filling the numbers $1,2, \ldots, m+n$ in a given border strip $\left\langle k_{1}, k_{2}, \ldots, k_{r}\right\rangle$ by the rules:

- Entries in each row are increasing, allowing the repetition of elements in $\left\{i \mid i \in \mathbf{B}_{+}^{(m)}\right\}$, but not permitting the repetition of elements in $\left\{i \mid i \in \mathbf{B}_{-}^{(n)}\right\}$,

- Entries in each column are increasing, allowing the repetition of elements in $\left\{i \mid i \in \mathbf{B}_{-}^{(n)}\right\}$, but not permitting the repetition of elements in $\left\{i \mid i \in \mathbf{B}_{+}^{(m)}\right\}$. 
Let $\mathcal{G}_{\left\langle k_{1}, k_{2}, \ldots, k_{r}\right\rangle}^{(m \mid n)}$ be the set of all Young tableaux which are obtained by filling up the border strip $\left\langle k_{1}, k_{2}, \ldots, k_{r}\right\rangle$ through the above mentioned rules. The super Schur polynomial corresponding to $\left\langle k_{1}, k_{2}, \ldots, k_{r}\right\rangle$ is then defined as

$$
S_{\left\langle k_{1}, k_{2}, \ldots, k_{r}\right\rangle}(x, y)=\sum_{T \in \mathcal{G}_{\left\langle k_{1}, k_{2}, \ldots, k_{r}\right\rangle}^{(m \mid n)}} e^{\mathrm{wt}(T)} .
$$

Here the weight $\mathrm{wt}(T)$ of the Young tableaux $T$ is given by

$$
\mathrm{wt}(T)=\sum_{\alpha=1}^{m+n} \alpha(T) \epsilon_{\alpha},
$$

where $\alpha(T)$ denotes the multiplicity of the number $\alpha$ in the Young Tableaux $T$, and we use the notations

$$
x_{\alpha} \equiv e^{\epsilon_{\alpha}} \quad \text { for } \quad \alpha \in \mathbf{B}_{+}^{(m)}, \quad y_{\alpha-m} \equiv e^{\epsilon_{\alpha}} \quad \text { for } \quad \alpha \in \mathbf{B}_{-}^{(n)} .
$$

The dimensionality of the irreducible representation associated with the border strip $\left\langle k_{1}, k_{2}, \ldots\right.$, $\left.k_{r}\right\rangle$ can be found by setting $x=1, y=1$ in the corresponding Schur polynomial $S_{\left\langle k_{1}, k_{2}, \ldots, k_{r}\right\rangle}(x, y)$. Hence, by using equation (2.2), we obtain the dimension of irreducible representation as

$$
\left.S_{\left\langle k_{1}, k_{2}, \ldots, k_{r}\right\rangle}(x, y)\right|_{x=1, y=1}=\mathcal{N}_{\left\langle k_{1}, k_{2}, \ldots, k_{r}\right\rangle}^{(m \mid n)},
$$

where $\mathcal{N}_{\left\langle k_{1}, k_{2}, \ldots, k_{r}\right\rangle}^{(m \mid n)}$ denotes the number of all allowed tableaux corresponding to the border strip $\left\langle k_{1}, k_{2}, \ldots, k_{r}\right\rangle$. For example, in the case of the $s u(2 \mid 1)$ spin chain, it is possible to construct the following tableaux corresponding to the border strip $\langle 2,1\rangle$ :

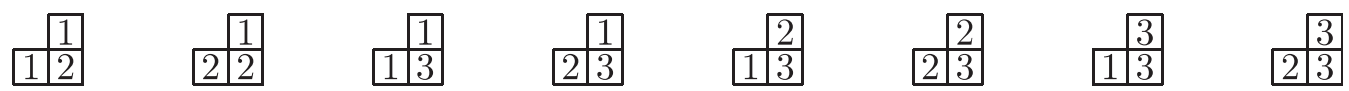

which gives $\mathcal{N}_{\langle 2,1\rangle}^{(2 \mid 1)}=8$.

It is worth noting that the border strips can equivalently be described by 'motifs' [18, 23], which for a spin chain with $N$ number of lattice sites is given by a sequence of $N-1$ number of 0 's and 1's, $\delta=\left(\delta_{1}, \delta_{2}, \ldots, \delta_{N-1}\right)$ with $\delta_{j} \in\{0,1\}$. In fact, there exists a one-to-one map from a border strip to a motif as

$$
\left\langle k_{1}, k_{2}, \ldots, k_{r}\right\rangle \Longrightarrow \delta=(\underbrace{1, \ldots, 1}_{k_{1}-1}, 0, \underbrace{1, \ldots, 1}_{k_{2}-1}, 0, \ldots \ldots, 0, \underbrace{1, \ldots, 1}_{k_{r}-1}) .
$$

For example, the border strip $\langle 2,3,1\rangle$ is mapped to the motif (10110). Let us now define the partial sums corresponding to the border strip $\left\langle k_{1}, k_{2}, \ldots, k_{r}\right\rangle$ as

$$
\kappa_{i}=\sum_{l=1}^{i} k_{l}
$$

where $i \in\{1,2, \ldots, r-1\}$. By using equation (2.5) it is easy to see that, the elements of motif $\delta$ satisfy the following rule: $\delta_{j}=0$ if $j$ coincides with one of the partial sums $\kappa_{i}$, and $\delta_{j}=1$ otherwise. The inverse mapping from a motif to a border strip can be obtained by reading a motif $\delta=\left(\delta_{1}, \delta_{2}, \ldots, \delta_{N-1}\right)$ from the left, and adding a box under (resp. left) the box when $\delta_{j}=1$ (resp. $\left.\delta_{j}=0\right)$ is encountered. 


\section{Equivalence between HS like spin chains and some vertex models}

\subsection{Definition of HS like spin chains and related vertex models}

Let us assume that their exists a class of $Y\left(s l_{(m \mid n)}\right)$ Yangian invariant HS like spin chains with $N$ number of lattice sites, for which the complete set of energy levels associated with border strips like $\left\langle k_{1}, k_{2}, \ldots, k_{r}\right\rangle$ (for all possible $\mathbf{k} \in \mathcal{P}_{N}$ ) can be written in the form

$$
E_{\left\langle k_{1}, k_{2}, \ldots, k_{r}\right\rangle}=\sum_{j=1}^{r-1} \mathcal{E}_{N}\left(\kappa_{j}\right),
$$

where $\mathcal{E}_{N}(j)$ is an arbitrary function of the discrete variable $j \in\{1,2, \ldots, N-1\}$. Since any Yangian invariant spin chain must have the same energy eigenvalue corresponding to all eigenfunctions which form an irreducible representation of the $Y\left(s l_{(m \mid n)}\right)$ algebra, the multiplicity of the eigenvalue $E_{\left\langle k_{1}, k_{2}, \ldots, k_{r}\right\rangle}$ in equation (3.1) would be given by $\mathcal{N}_{\left\langle k_{1}, k_{2}, \ldots, k_{r}\right\rangle}^{(m \mid n)}$. Therefore, the partition function of such a spin chain can be expressed as

$$
Z_{N}^{(m \mid n)}(q)=\sum_{\mathbf{k} \in P_{N}} q^{\sum_{j=1}^{r-1} \mathcal{E}_{N}\left(\kappa_{j}\right)} \mathcal{N}_{\left\langle k_{1}, k_{2}, \ldots, k_{r}\right\rangle}^{(m \mid n)}
$$

where $q=e^{-\frac{1}{k T}}$. We also define a 'generalized partition function' for this spin chain as

$$
Z_{N}^{(m \mid n)}(q ; x, y)=\sum_{\mathbf{k} \in P_{N}} q^{\sum_{j=1}^{r-1} \mathcal{E}_{N}\left(\kappa_{j}\right)} S_{\left\langle k_{1}, k_{2}, \ldots, k_{r}\right\rangle}(x, y),
$$

which reduces to the usual partition function (3.2) in the special case $x=1, y=1$. It is worth noting that, all eigenvalues of the Hamiltonians (1.1) and (1.2) can be written in the form (3.1) with $\mathcal{E}_{N}(j)=j(N-j)$ and $\mathcal{E}_{N}(j)=j$ respectively [21, 26]. Hence, both of the $s u(m \mid n)$ supersymmetric HS and Polychronakos spin chains belong to the above defined class of Yangian invariant spin systems. Furthermore, the results of [29] imply that the energy levels of a hyperbolic version of the HS spin chain, known as Frahm-Inozemstsev chain [30], can also be expressed in the form (3.1) at least in the nonsupersymmetric limit. We shall discuss about this spin chain in more detail in the Subsection 3.3. So there exist quite a few quantum spin chains with long range interaction, whose energy levels can be expressed through equation (3.1). However, as far as we know, there exists no general method for constructing the Hamiltonian of a quantum spin chain in terms of the generators of $s u(m \mid n)$ algebra, such that the corresponding energy levels are expressed through equation (3.1) for a given functional form of $\mathcal{E}_{N}(j)$.

Next, we consider a class of $1 \mathrm{D}$ classical vertex models with $(N+1)$ number of vertices, which are connected through $N$ number of intermediate bonds. Each of these bonds can take either one of the $m$ possible bosonic states or one of the $n$ possible fermionic states. Any possible state for such $s u(m \mid n)$ vertex model can be represented by a path configuration of finite length given by

$$
\vec{s} \equiv\left\{s_{1}, s_{2}, \ldots, s_{N}\right\},
$$

where $s_{i} \in\{1,2, \ldots, m+n\}$ denotes the spin state of the $i$-th bond. Let us define an 'energy function' associated with the spin path configuration $\vec{s}$ as

$$
E^{(m \mid n)}(\vec{s})=\sum_{j=1}^{N-1} \mathcal{E}_{N}(j) H^{(m \mid n)}\left(s_{j}, s_{j+1}\right),
$$


where

$$
H^{(m \mid n)}\left(s_{j}, s_{j+1}\right)=\left\{\begin{array}{ll}
0 & \text { if } s_{j}<s_{j+1} \text { or } s_{j}=s_{j+1} \in \mathbf{B}_{-}^{(n)}, \\
1 & \text { if } s_{j}>s_{j+1} \text { or } s_{j}=s_{j+1} \in \mathbf{B}_{+}^{(m)}
\end{array},\right.
$$

and $\mathcal{E}_{N}(j)$ is an arbitrary function of the discrete variable $j$, which has also appeared in equation (3.1). The form of energy function (3.5) implies that the extreme left and extreme right vertices, which are connected through only one bond, always have the zero energy. The energy of all other vertices, which are connected by two bonds, may have a finite nonzero value. For example, the energy of the $j$-th vertex is given by

$$
E_{j}^{(m \mid n)}\left(s_{j-1}, s_{j}\right)=\tilde{\mathcal{E}}_{N}(j) H^{(m \mid n)}\left(s_{j-1}, s_{j}\right),
$$

where $\tilde{\mathcal{E}}_{N}(j) \equiv \mathcal{E}_{N}(j-1)$ and $j \in\{2,3, \ldots, N\}$. The energy function (3.5) is obtained by summing up these nontrivial vertex energies as

$$
E^{(m \mid n)}(\vec{s})=\sum_{j=2}^{N} E_{j}^{(m \mid n)}\left(s_{j-1}, s_{j}\right)
$$

The partition function for this type of inhomogeneous $s u(m \mid n)$ vertex model may be written as

$$
V_{N}^{(m \mid n)}(q)=\sum_{\vec{s}} q^{E^{(m \mid n)}(\vec{s})}
$$

where the summation has been taken for all possible path configurations. In analogy with the case of Young tableaux, one can construct a weight function for the spin path configuration $\vec{s}$ as

$$
\mathrm{wt}(\vec{s})=\sum_{\alpha=1}^{m+n} \alpha(\vec{s}) \epsilon_{\alpha}
$$

where $\alpha(\vec{s})$ denotes the multiplicity of the spin component $\alpha$ in the path configuration $\vec{s}$. By using such weight functions and the notations given in equation (2.4), we define a generalized partition function or character for the vertex model as

$$
V_{N}^{(m \mid n)}(q ; x, y)=\sum_{\vec{s}} q^{E^{(m \mid n)}(\vec{s})} e^{\mathrm{wt}(\vec{s})}
$$

It is evident that this expression for generalized partition function reduces to (3.7) in the special case $x=1, y=1$. Let us now propose an equality between the generalized partition function of the HS like spin chain and that of the $1 \mathrm{D}$ vertex model as

$$
Z_{N}^{(m \mid n)}(q ; x, y)=V_{N}^{(m \mid n)}(q ; x, y) .
$$

We would like to make some comments at present. The equality (3.10) has been established earlier in the case of $s u(m)$ and $s u(m \mid n)$ Polychronakos spin chains for which $\mathcal{E}_{N}\left(\kappa_{j}\right)=\kappa_{j}$ $[27,28]$. In the following Subsection 3.2, however, we shall prove the relation (3.10) without assuming any particular form of the function $\mathcal{E}_{N}\left(\kappa_{j}\right)$. Next, in Subsection 3.3, we shall express the $x=y=1$ limit of equality (3.10) in an alternative form. Using some results of our previous works [26, 31], such alternative form of equality (3.10) has been guessed recently for the case of nonsupersymmetric HS like spin chains [32]. 


\subsection{Proof of the equivalence relation}

At first, we notice that one can map spin path configurations associated with vertex models to the motifs associated with Yangian invariant spin chains by using some well defined rules [27, $28,32]$. For our purpose, we take a slightly modified version of this mapping, which maps the spin path configuration $\vec{s}=\left\{s_{1}, s_{2}, \ldots, s_{N}\right\}$ to the motif $\delta=\left(\delta_{1}, \delta_{2}, \ldots, \delta_{N-1}\right)$ by using the rules

(i) if $s_{i}<s_{i+1}$ or $s_{i}=s_{i+1} \in \mathbf{B}_{-}^{(n)}$, then $\delta_{i}=1$,

(ii) if $s_{i}>s_{i+1}$ or $s_{i}=s_{i+1} \in \mathbf{B}_{+}^{(m)}$, then $\delta_{i}=0$.

Note that the above mentioned mapping from a spin configuration to a motif is not one-to-one. For example, in the particular case of $s u(2 \mid 0)$ model with $N=3$, both of the spin configurations $\{112\}$ and $\{212\}$ are mapped to the same motif (01). Now we consider two consecutive lemmas which will be instrumental in proving the relation (3.10).

Lemma 1. Let us assume that a spin configuration $\vec{s} \equiv\left\{s_{1}, s_{2}, \ldots, s_{N}\right\}$ is mapped to the motif $\delta \equiv\left(\delta_{1}, \delta_{2}, \ldots, \delta_{N-1}\right)$ by using the rules given in (3.11), and this motif $\delta$ is subsequently mapped to the border strip $\left\langle k_{1}, k_{2}, \ldots, k_{r}\right\rangle$ by using the inverse of the mapping defined in equation (2.5). Then the sequence of numbers $\left\{s_{1}, s_{2}, \ldots, s_{N}\right\}$ would generate an allowed tableaux for the border strip $\left\langle k_{1}, k_{2}, \ldots, k_{r}\right\rangle$, where the spin $s_{i}$ is put in the $i$-th box starting from the uppermost right side of the border strip.

Proof. We put the spin $s_{i}$ in the $i$-th box and spin $s_{i+1}$ in the $(i+1)$-th box starting from the uppermost right side of the border strip. The relative positions of these boxes can be either

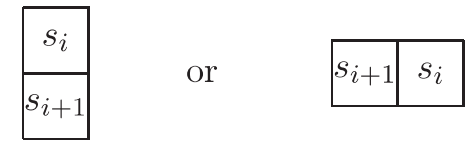

Let us first assume that either $s_{i}<s_{i+1}$ or $s_{i}=s_{i+1} \in \mathbf{B}_{-}^{(n)}$, which leads to $\delta_{i}=1$ by applying rule (i) in equation (3.11). According to the inverse of the mapping defined in equation (2.5), the $(i+1)$-th box should be placed below the $i$-th box if $\delta_{i}=1$ :

$$
\begin{aligned}
& i \text {-th box } \\
& (i+1) \text {-th box }
\end{aligned}
$$

Therefore, for either $s_{i}<s_{i+1}$ or $s_{i}=s_{i+1} \in \mathbf{B}_{-}^{(n)}$, we get a part of tableaux where the $i$-th box and $(i+1)$-th box are positioned as

$$
\begin{array}{|c|}
\hline s_{i} \\
\hline s_{i+1} \\
\hline
\end{array}
$$

It should be observed that, according to the rules of constructing a Young tableaux which have been mentioned just after equation (2.1), the above figure represents an allowed configuration inside a border strip.

Next, we examine the remaining possibility, i.e. either $s_{i}>s_{i+1}$ or $s_{i}=s_{i+1} \in \mathbf{B}_{+}^{(m)}$, which yields $\delta_{i}=0$ by applying rule (ii) in equation (3.11). According to the inverse of the mapping defined in equation (2.5), the $(i+1)$-th box should be placed on the left side of the $i$-th box if $\delta_{i}=0$ :

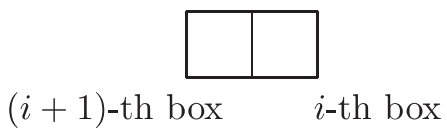


Thus for either $s_{i}>s_{i+1}$ or $s_{i}=s_{i+1} \in \mathbf{B}_{+}^{(m)}$, we get a part of tableaux where the $i$-th box and $(i+1)$-th box are positioned as

$$
\begin{array}{|l|l|}
\hline s_{i+1} & s_{i} \\
\hline
\end{array}
$$

Again, it may be noted that, according to the rules of constructing a Young tableaux which have been mentioned just after equation (2.1), the above figure represents an allowed configuration inside a border strip. Hence we find that, for all possible choice of $s_{i}$ and $s_{i+1}$, the $i$-th and $(i+1)$-th box form a part of an allowed tableaux. Applying the aforesaid prescription repeatedly, we can easily show that the spin configuration $\left\{s_{1}, s_{2}, \ldots, s_{N}\right\}$ generates an allowed tableaux for the associated border strip $\left\langle k_{1}, k_{2}, \ldots, k_{r}\right\rangle$.

It is evident that, due to Lemma 1, there exists a one-to-one mapping from the set of all spin path configurations of the form (3.4) to the set of Young tableaux which are constructed by filling up all possible border strips like $\left\langle k_{1}, k_{2}, \ldots, k_{r}\right\rangle$, where $\mathbf{k} \in \mathcal{P}_{N}$. It is trivial to construct the inverse of this one-to-one mapping, which maps a Young tableaux to a spin path configuration. By comparing equations (2.3) and (3.8), we find that the weight of a Young tableaux $T$ coincides with the weight of the corresponding spin path configuration $\vec{s}$ :

$$
\mathrm{wt}(T)=\operatorname{wt}(\vec{s})
$$

Let $\mathcal{F}_{\left\langle k_{1}, k_{2}, \ldots, k_{r}\right\rangle}^{(m \mid n)}$ be the set of all path configurations which are mapped to a given border strip $\left\langle k_{1}, k_{2}, \ldots, k_{r}\right\rangle$ by successively applying the mapping defined in equation (3.11) and the inverse of the mapping defined in equation (2.5). Due to Lemma 1, there exists a one-to-one correspondence between the sets $\mathcal{F}_{\left\langle k_{1}, k_{2}, \ldots, k_{r}\right\rangle}^{(m \mid n)}$ and $\mathcal{G}_{\left\langle k_{1}, k_{2}, \ldots, k_{r}\right\rangle}^{(m \mid n)}$. Consequently, by using equations (2.2) and (3.12), one can express the super Schur polynomial corresponding to the border strip $\left\langle k_{1}, k_{2}, \ldots, k_{r}\right\rangle$ as

$$
S_{\left\langle k_{1}, k_{2}, \ldots, k_{r}\right\rangle}(x, y)=\sum_{\vec{s} \in \mathcal{F}_{\left\langle k_{1}, k_{2}, \ldots, k_{r}\right\rangle}^{(m \mid n)}} e^{\mathrm{wt}(\vec{s})} .
$$

Now, we consider the second lemma:

Lemma 2. If the spin configuration $\vec{s} \equiv\left\{s_{1}, s_{2}, \ldots, s_{N}\right\}$ is mapped to the border strip $\left\langle k_{1}, k_{2}, \ldots\right.$, $\left.k_{N}\right\rangle$ by successively applying the mapping defined in equation (3.11) and the inverse of the mapping defined in equation (2.5), then the energy function (3.5) associated with $\vec{s}$ can be expressed as

$$
E^{(m \mid n)}(\vec{s})=\sum_{l=1}^{r-1} \mathcal{E}_{N}\left(\kappa_{l}\right) .
$$

Proof. By comparing equation (3.6) with equation (3.11), one can write $H^{(m \mid n)}\left(s_{j}, s_{j+1}\right)$ as

$$
H^{(m \mid n)}\left(s_{j}, s_{j+1}\right)=1-\delta_{j}
$$

Substituting this expression of $H^{(m \mid n)}\left(s_{j}, s_{j+1}\right)$ to equation (3.5), we obtain

$$
E^{(m \mid n)}(\vec{s})=\sum_{j=1}^{N-1} \mathcal{E}_{N}(j)\left(1-\delta_{j}\right)
$$

Thus, only the zeros of the motif $\left(\delta_{1}, \delta_{2}, \ldots, \delta_{N-1}\right)$ would contribute to $E^{(m \mid n)}(\vec{s})$. We have already mentioned that in Section 2 that, the positions of the zeros of a motif coincide with the 
partial sums (2.6) associated with the corresponding border strip $\left\langle k_{1}, k_{2}, \ldots, k_{r}\right\rangle$. Therefore, we can write $\left(1-\delta_{j}\right)$ in terms of Kronecker $\delta$-function as

$$
1-\delta_{j}=\sum_{l=1}^{r-1} \delta_{j, \kappa_{l}}
$$

Substituting this expression of $\left(1-\delta_{j}\right)$ to equation (3.15), and summing over the index $j$ by using Kronecker $\delta$-function, we easily get equation (3.14). The form of equation (3.14) ensures that, for all spin path configurations $\left\{s_{1}, s_{2}, \ldots, s_{N}\right\} \in \mathcal{F}_{\left\langle k_{1}, k_{2}, \ldots, k_{r}\right\rangle}^{(m \mid n)}$, we get the same value of the energy function.

Now we are in a position to prove the main proposition of this paper, which is given in equation (3.10). To this end we note that, as a consequence of Lemma 1, equation (3.9) can be written by rearranging its summation variables as

$$
V_{N}^{(m \mid n)}(q ; x, y)=\sum_{\mathbf{k} \in P_{N}} \sum_{\vec{s} \in \mathcal{F}_{\left\langle k_{1}, k_{2}, \ldots, k_{r}\right\rangle}^{(m \mid n)}} q^{E^{(m \mid n)}(\vec{s})} e^{\mathrm{wt}(\vec{s})} .
$$

Substituting the expression of $E^{(m \mid n)}(\vec{s})$ given in equation (3.14) to equation (3.16), we find that

$$
V_{N}^{(m \mid n)}(q ; x, y)=\sum_{\mathbf{k} \in P_{N}} q^{\sum_{l=1}^{r-1} \mathcal{E}_{N}\left(\kappa_{l}\right)} \sum_{\vec{s} \in \mathcal{F}_{\left\langle k_{1}, k_{2}, \ldots, k_{r}\right\rangle}^{(m \mid n)}} e^{\mathrm{wt}(\vec{s})} .
$$

Finally, by using the relation (3.13), we can express equation (3.17) as

$$
V_{N}^{(m \mid n)}(q ; x, y)=\sum_{\mathbf{k} \in P_{N}} q^{\sum_{l=1}^{r-1} \mathcal{E}_{N}\left(\kappa_{l}\right)} S_{\left\langle k_{1}, k_{2}, \ldots, k_{r}\right\rangle}(x, y) .
$$

Since the r.h.s. of equation (3.18) coincide with that of equation (3.3), we readily obtain a proof of equation (3.10). By putting $x=y=1$ in equation (3.10), we also get

$$
Z_{N}^{(m \mid n)}(q)=V_{N}^{(m \mid n)}(q)
$$

which shows that the partition functions of all Yangian invariant HS like spin chains, with energy levels given by equation (3.1), exactly coincide with the partition functions of $1 \mathrm{D}$ vertex models with energy functions given by equation (3.5). Moreover, since $Z_{N}^{(m \mid n)}(q)$ or $V_{N}^{(m \mid n)}(q)$ can be expressed as a power series of $q$, where the powers represent all possible energy levels or energy functions, it is clear from the equality (3.19) that the energy levels of the HS like spin chains exactly match with the energy functions of the corresponding vertex models.

We have already mentioned that, all eigenvalues of the Hamiltonians (1.1) and (1.2) can be written in the form (3.1), with $\mathcal{E}_{N}(j)=j(N-j)$ and $\mathcal{E}_{N}(j)=j$ respectively. Hence, due to equation (3.19) it follows that, the partition functions of $s u(m \mid n)$ supersymmetric HS and Polychronakos spin chains would be equivalent to those of $s u(m \mid n)$ vertex models with energy functions given by (3.5), where $\mathcal{E}_{N}(j)=j(N-j)$ and $\mathcal{E}_{N}(j)=j$ respectively. It should be noted that, since the relations (3.10) and (3.19) have been derived by assuming that $m$ and $n$ are arbitrary non-negative integers (excluding the nonphysical case $m=n=0$ ), these relations are also valid for the nonsupersymmetric cases like $n=0, m>1$ or $m=0, n>1$. Therefore, by considering equation (3.19) for such special cases, one can also establish an equivalence between the partition functions of nonsupersymmetric HS like spin chains and those of the corresponding $1 \mathrm{D}$ vertex models. 


\subsection{An alternative form of the equivalence relation}

Let us now try to express the remarkable relation (3.19) in an alternative form. It is well known that the 'freezing trick' provides a powerful method of calculating the partition functions of quantum integrable spin chains with long range interaction [10]. By using this freezing trick, the partition functions of supersymmetric and nonsupersymmetric spin systems associated with the $A_{N}$ type of root system can often be expressed in the form [33, 24, 31, 34]

$$
\widetilde{Z}^{(m \mid n)}(q)=\sum_{\mathbf{k} \in \mathcal{P}_{N}} \prod_{i=1}^{r} d^{(m \mid n)}\left(k_{i}\right) \cdot q^{\sum_{j=1}^{r-1} \mathcal{E}\left(\kappa_{j}\right)} \prod_{i=1}^{N-r}\left(1-q^{\mathcal{E}\left(\kappa_{i}^{\prime}\right)}\right),
$$

where $\kappa_{1}^{\prime}, \kappa_{2}^{\prime}, \ldots, \kappa_{N-r}^{\prime}$ are the complements of the partial sums defined in equation (2.6), i.e. $\kappa_{1}^{\prime}, \kappa_{2}^{\prime}, \ldots, \kappa_{N-r}^{\prime} \equiv\{1,2, \ldots, N-1\}-\left\{\kappa_{1}, \kappa_{2}, \ldots, \kappa_{r-1}\right\}$, and $d^{(m \mid n)}\left(k_{i}\right)$ is given by

$$
d^{(m \mid n)}\left(k_{i}\right)= \begin{cases}{ }^{m} C_{k_{i}}, & \text { if } n=0, m>1, \\ { }^{n+k_{i}-1} C_{k_{i}}, & \text { if } m=0, n>1, \\ \sum_{j=0}^{\min \left(m, k_{i}\right)}{ }^{m} C_{j}{ }^{k_{i}-j+n-1} C_{k_{i}-j}, & \text { if } m, n>0,\end{cases}
$$

with ${ }^{p} C_{l} \equiv \frac{p !}{l(p-l) !}$ for $l \leq p$ and ${ }^{p} C_{l} \equiv 0$ for $l>p$. It can be shown that, for any possible choice of the function $\mathcal{E}(j)$, the partition functions given by equations (3.2) and (3.20) are completely equivalent to each other $[26,31]$ :

$$
Z^{(m \mid n)}(q)=\widetilde{Z}^{(m \mid n)}(q)
$$

Due to this equivalence, we can express equation (3.19) in an alternative form like

$$
\widetilde{Z}^{(m \mid n)}(q)=V^{(m \mid n)}(q) .
$$

It should be noted that, the nonsupersymmetric version of the above relation has been stated recently (without an explicit proof) and also used to analytically find out the level density distribution of some HS like spin chains with large number of lattice sites [32].

In this context we would like to mention that, there exist a hyperbolic variant [30] of the HS spin chain, whose partition function has been calculated for the nonsupersymmetric case by using the freezing trick [29]. The Hamiltonian of such hyperbolic version of the HS spin, known as Frahm-Inozemstsev (FI) chain, can be written for the most general $s u(m \mid n)$ supersymmetric case as

$$
\mathcal{H}_{\mathrm{FI}}^{(m \mid n)}=\frac{1}{2} \sum_{j<k} \frac{1+\hat{P}_{j k}^{(m \mid n)}}{\sinh ^{2}\left(\xi_{j}-\xi_{k}\right)},
$$

where the lattice sites of this chain are given by $\xi_{i}=\frac{1}{2} \ln \zeta_{i}$, and $\zeta_{i}$ is the $i$-th zero of the Laguerre polynomial $L_{N}^{\alpha-1}$ with $\alpha>0$. It has been found that, for nonsupersymmetric cases like $n=0, m>1$ and $m=0, n>1$, partition functions of this spin chain can be obtained in the form (3.20), with $\mathcal{E}(j)=j(\alpha+j-1)$ [29]. Hence, by using equation (3.21), one can show that the energy levels of nonsupersymmetric FI spin chains are expressed through equation (3.1) with exactly same form of $\mathcal{E}(j)$. Moreover, due to the relation (3.22), it is evident that the corresponding partition functions would be equivalent to those of $s u(m \mid 0)$ and $s u(0 \mid n)$ vertex models with energy functions given by equation (3.5), where $\mathcal{E}(j)=j(\alpha+j-1)$. 


\subsection{Boson-fermion duality relation}

Let us now discuss an interesting application of the above mentioned equivalence between the partition functions of HS like spin chains and 1D vertex models. It is known that the partition functions of the $s u(m \mid n)$ supersymmetric HS and Polychronakos spin chains satisfy a remarkable duality relation under the exchange of bosonic and fermionic spin degrees of freedom [25, 21, 24, 26, 23]. Here, we want to show that the partition functions of all HS like spin chains, which can be expressed in the form (3.2) or (3.20), would satisfy this type of boson-fermion duality relation. To this end, let us define a mapping which maps a spin path configuration $\vec{s} \equiv\left\{s_{1}, s_{2}, \ldots, s_{N}\right\}$ of $s u(m \mid n)$ vertex model to a spin path configuration $\vec{t} \equiv\left\{t_{1}, t_{2}, \ldots, t_{N}\right\}$ of $s u(n \mid m)$ vertex model as

$$
t_{i}=(m+n+1)-s_{i},
$$

where $i \in\{1,2, \ldots, N\}$. Note that (3.23) represents a one-to-one and invertible mapping from the set of all spin path configurations of $s u(m \mid n)$ vertex model to the set of all spin path configurations of $s u(n \mid m)$ vertex model. Moreover, it may also be observed that, if $s_{i} \in B_{+}^{(m)}$ (resp. $s_{i} \in B_{-}^{(n)}$ ) then $t_{i} \in B_{-}^{(m)}$ (resp. $t_{i} \in B_{+}^{(n)}$ ), and if $s_{i}<s_{i+1}\left(\right.$ resp. $\left.s_{i}>s_{i+1}\right)$ then $t_{i}>t_{i+1}$ (resp. $t_{i}<t_{i+1}$ ). Hence, due to equation (3.6), it follows that

$$
H^{(m \mid n)}\left(s_{j}, s_{j+1}\right)=1-H^{(n \mid m)}\left(t_{j}, t_{j+1}\right) .
$$

Substituting the above relation to equation (3.5), we obtain

$$
E^{(m \mid n)}(\vec{s})=\Omega-E^{(n \mid m)}(\vec{t})
$$

where $\Omega$ is given by

$$
\Omega=\sum_{j=1}^{N-1} \mathcal{E}_{N}(j) .
$$

By using equations (3.7) and (3.24), we find that there exists a boson-fermion duality relation between the partition functions of $s u(m \mid n)$ and $s u(n \mid m)$ vertex models:

$$
V^{(m \mid n)}(q)=q^{\Omega} V^{(n \mid m)}\left(q^{-1}\right)
$$

Due to the equality (3.19), the above equation can be transformed to a boson-fermion duality relation between the partition functions of $s u(m \mid n)$ and $s u(n \mid m)$ spin chains as

$$
Z^{(m \mid n)}(q)=q^{\Omega} Z^{(n \mid m)}\left(q^{-1}\right)
$$

Thus we are able to prove that, the partition functions of all HS like spin chains, which can be expressed in the form (3.2), would satisfy the duality relation (3.26) where $\Omega$ is obtained by using equation (3.25). Due to equation (3.22) it follows that, the partition function $\widetilde{Z}^{(m \mid n)}(q)$ given in equation (3.20) would also satisfy the exactly same form of duality relation. Since the partition functions of $s u(m \mid n)$ HS and Polychronakos spin chains can be written in the form (3.2) with $\mathcal{E}_{N}(j)=j(N-j)$ and $\mathcal{E}_{N}(j)=j$ respectively, it is evident that these partition functions satisfy the boson-fermion duality relation (3.26) with $\Omega=\sum_{j=1}^{N-1} j(N-j)=N\left(N^{2}-1\right) / 6$ and $\Omega=\sum_{j=1}^{N-1} j=N(N-1) / 2$ respectively. 


\section{Concluding remarks}

In this article we consider a class of $Y\left(s l_{(m \mid n)}\right)$ Yangian invariant spin chains, which contain the well known $s u(m \mid n)$ supersymmetric Haldane-Shastry (HS) and Polychronakos spin chains as special cases. Since the irreducible representations of the $Y\left(s l_{(m \mid n)}\right)$ algebra can be characterized through the border strips, partition functions of this class of HS like spin chains can be expressed in terms of the super Schur polynomials associated with the border strips. Representing such super Schur polynomial as a summation over spin path configurations, we establish the key relations (3.10) and (3.19), which show that the partition functions of this class of spin chains are equivalent to the partition functions of a class of one-dimensional (1D) vertex models with appropriately defined energy functions. Since $s u(m \mid n)$ supersymmetric HS and Polychronakos spin chains belong to this class of Yangian invariant spin chains, we easily obtain the corresponding vertex models through this approach. Moreover, the vertex model associated with FI spin chain can also be obtained through this approach, at least in the nonsupersymmetric case.

In this context it is interesting to recall that, there exists a close connection between 1D quantum integrable spin chains with short range interaction and $2 \mathrm{D}$ vertex models in classical statistical mechanics $[4,5]$. However, for these cases, there exists no simple relation between the energy levels of 1D spin chains and the energy functions of $2 \mathrm{D}$ vertex models. On the other hand, the presently derived relation (3.19) implies that, all energy levels of a class of HS like spin chains with long range interaction exactly coincide (including the degeneracy factors) with the energy functions of some 1D inhomogeneous vertex models in classical statistical mechanics. Hence, this connection between 1D quantum spin chains and 1D classical vertex models is much more direct in nature. By using this connection, we have derived a boson-fermion duality relation (3.26) for the partition functions of this class of supersymmetric HS like spin chains. Moreover, it may be noted that, the nonsupersymmetric limit of this connection has been used very recently to analytically find out the level density distribution of some bosonic and fermionic HS like spin chains [32]. Therefore, it is natural to expect that the more general equivalence relation (3.19) would also be useful to analytically find out the level density distribution of supersymmetric HS like spin chains. Finally, this type of connection between HS like spin chains with long range interaction and 1D vertex models may also be useful in calculating various correlation functions and statistical properties of these systems.

\section{References}

[1] Sutherland B., Beautiful models. 70 years of exactly solved quantum many-body problems, World Scientific Publishing Co., Inc., River Edge, NJ, 2004.

[2] Korepin V.E., Bogoliubov N.M., Izergin A.G., Quantum inverse scattering method and correlation functions, Cambridge Monographs on Mathematical Physics, Cambridge University Press, Cambridge, 1993.

[3] Essler F.H.L., Frahm H., Göhmann F., Klümper A., Korepin V.E., The one-dimensional Hubbard model, Cambridge University Press, Cambridge, 2005.

[4] Sutherland B., Two-dimensional hydrogen bonded crystals without the ice rule, J. Math. Phys. 11 (1970), 3183-3186.

[5] Baxter R.J., One-dimensional anisotropic Heisenberg chain, Phys. Rev. Lett. 26 (1971), 834-834.

[6] Haldane F.D.M., Exact Jastrow-Gutzwiller resonating-valence-bond ground state of the spin- $1 / 2$ antiferromagnetic Heisenberg chain with $1 / r^{2}$ exchange, Phys. Rev. Lett. 60 (1988), 635-638.

[7] Shastry B.S., Exact solution of an $S=1 / 2$ Heisenberg antiferromagnetic chain with long-ranged interactions, Phys. Rev. Lett. 60 (1988), 639-642.

[8] Polychronakos A.P., Lattice integrable systems of Haldane-Shastry type, Phys. Rev. Lett. 70 (1993), 23292331, hep-th/9210109.

[9] Frahm H., Spectrum of a spin chain with inverse square exchange, J. Phys. A: Math. Gen. 26 (1993), L473-L479, cond-mat/9303050. 
[10] Polychronakos A.P., Exact spectrum of $S U(n)$ spin chain with inverse-square exchange, Nuclear Phys. B 419 (1994), 553-566.

[11] Haldane F.D.M., "Fractional statistics" in arbitrary dimensions: a generalization of the Pauli principle, Phys. Rev. Lett. 67 (1991), 937-940.

[12] Ha Z.N.C., Quantum many-body systems in one dimension, Series on Advances in Statistical Mechanics, Vol. 12, World Scientific Publishing Co., Inc., River Edge, NJ, 1996.

[13] Bernevig B.A., Gurarie V., Simon S.H., Central charge and quasihole scaling dimensions from model wavefunctions: towards relating Jack wavefunctions to $W$-algebras, J. Phys. A: Math. Theor. 42 (2009), 245206, 30 pages, arXiv:0903.0635.

[14] Bernevig B. A., Haldane F.D.M., Clustering properties and model wavefunctions for non-Abelian fractional quantum Hall quasielectrons, Phys. Rev. Lett. 102 (2009), 066802, 4 pages, arXiv:0810.2366.

[15] Beisert N., Dippel V., Staudacher M., A novel long-range spin chain and planar $\mathcal{N}=4$ super Yang-Mills, J. High Energy Phys. 2004 (2004), no. 7, 075, 49 pages, hep-th/0405001.

[16] Serban D., Staudacher M., Planar $\mathcal{N}=4$ gauge theory and the Inozemtsev long range spin chain, J. High Energy Phys. 2004 (2004), no. 6, 001, 31 pages, hep-th/0401057.

[17] Beisert N., Staudacher M., Long-range psu(2,2|4) Bethe ansätze for gauge theory and strings, Nuclear Phys. B $\mathbf{7 2 7}$ (2005), 1-62, hep-th/0504190.

[18] Haldane F.D.M., Ha Z.N.C., Talstra J.C., Benard D., Pasquier V., Yangian symmetry of integrable quantum chains with long-range interactions and a new description of states in conformal field theory, Phys. Rev. Lett. 69 (1992), 2021-2025.

[19] Benard D., Gaudin M., Haldane F.D.M., Pasquier V., Yang-Baxter equation in long-range interacting systems, J. Phys. A: Math. Gen. 26 (1993), 5219-5236.

[20] Hikami K., Yangian symmetry and Virasoro character in a lattice spin system with long-range interactions, Nuclear Phys. B 441 (1995), 530-548.

[21] Hikami K., Basu-Mallick B., Supersymmetric Polychronakos spin chain: motif, distribution function, and character, Nuclear Phys. B 566 (2000), 511-528, math-ph/9904033.

[22] Bazhanov V.V., Frassek R., Lukowski T., Meneghelli C., Staudacher M., Baxter Q-operators and representations of Yangians, arXiv:1010.3699.

[23] Haldane F.D.M., Physics of the ideal semion gas: spinons and quantum symmetries of the integrable Haldane-Shastry spin chain, in Proc. 16th Taniguchi Symp. (Kashikojima, Japan, 1993), Editors A. Okiji and N. Kawakami, Springer, 1994, 3-20, cond-mat/9401001.

[24] Basu-Mallick B., Bondyopadhaya N., Exact partition function of $S U(m \mid n)$ supersymmetric Haldane-Shastry spin chain, Nuclear Phys. B 757 (2006), 280-302, hep-th/0607191.

[25] Basu-Mallick B., Ujino H., Wadati M., Exact spectrum and partition function of $S U(m \mid n)$ supersymmetric Polychronakos model, J. Phys. Soc. Japan 68 (1999), 3219-3226, hep-th/9904167.

[26] Basu-Mallick B., Bondyopadhaya N., Hikami K., Sen D., Boson-fermion duality in $S U(m \mid n)$ supersymmetric Haldane-Shastry spin chain, Nuclear Phys. B $\mathbf{7 8 2}$ (2007), 276-295, hep-th/0703240.

[27] Kirilov A.N., Kuniba A., Nakanishi T., Skew Young diagram method in spectral decomposition of integrable lattice models, Comm. Math. Phys. 185 (1997), 441-465, q-alg/9607027.

[28] Hikami K., Exclusion statistics and chiral partition function, in Physics and Combinatorics 2000 (Nagoya), Editors A.N. Kirilov and N. Liskova, World Sci. Publ., River Edge, NJ, 2001, 22-48.

[29] Barba J.C., Finkel F., González-López A., Rodríguez M.A., Inozemtsev's hyperbolic spin model and its related spin chain, Nuclear Phys. B 839 (2010), 499-525, arXiv:1005.0487.

[30] Frahm H., Inozemstsev V.I., New family of solvable 1D Heisenberg models, J. Phys. A: Math. Gen. 27 (1994), L801-L808, cond-mat/9405038.

[31] Basu-Mallick B., Bondyopadhaya N., Sen D., Low energy properties of the $S U(m \mid n)$ supersymmetric Haldane-Shastry spin chain, Nuclear Phys. B 795 (2008), 596-622, arXiv:0710.0452.

[32] Enciso A., Finkel F., González-López A., On the level density of spin chains of Haldane-Shastry type, arXiv:1005.3202.

[33] Finkel F., González-López A., Global properties of the spectrum of the Haldane-Shastry spin chain, Phys. Rev. B 72 (2005), 174411, 6 pages, cond-mat/0509032.

[34] Barba J.C., Finkel F., González-López A., Rodríguez M.A., The Berry-Tabor conjecture for spin chains of Haldane-Shastry type, Europhys. Lett. 83 (2008), 27005, 6 pages, arXiv:0804.3685. 